\title{
A phase II study of perioperative S-1 combined with weekly docetaxel in patients with locally advanced gastric carcinoma: clinical outcomes and clinicopathological and pharmacogenetic predictors for survival
}

\author{
Young-Woo Kim ${ }^{1} \cdot$ Mi-Jung Kim ${ }^{1} \cdot$ Keun Won Ryu ${ }^{1}$ Hyeong-Seok Lim ${ }^{2,3}$ • \\ Jun Ho Lee ${ }^{1} \cdot$ Sun-Young Kong ${ }^{2} \cdot J^{\prime}$ Song Seok Lee ${ }^{1}$ - Il Ju Choi ${ }^{1}$ Chan Gyoo Kim ${ }^{1}$. \\ Jong Yeul Lee ${ }^{1} \cdot$ Soo-Jeong Cho ${ }^{1} \cdot$ Myeong-Cherl Kook $^{1} \cdot$ Young-Iee Park ${ }^{1}$. \\ Seok-Ki Kim ${ }^{4}$ - Sook Ryun Park ${ }^{1,5}$ \\ Received: 21 October 2014/Accepted: 13 March 2015/Published online: 8 April 2015 \\ (c) The International Gastric Cancer Association and The Japanese Gastric Cancer Association 2015
}

\begin{abstract}
Background We conducted a phase II study to evaluate the efficacy and safety of perioperative S-1 plus docetaxel in locally advanced gastric cancer (LAGC) and to investigate the association between $C Y P 2 A 6$ genotype and outcome.

Methods Patients with LAGC [clinical stage III-IV (M0) by the Japanese staging system] received three cycles of pre- and postoperative chemotherapy ( $\mathrm{S}-140 \mathrm{mg} / \mathrm{m}^{2}$ twice daily on days $1-14$; intravenous docetaxel $35 \mathrm{mg} / \mathrm{m}^{2}$ on days 1 and 8 , every 3 weeks) followed by gastrectomy with D2 dissection. We also performed a pharmacokinetic and CYP2A6 genotyping study $(* 1, * 4, * 7, * 9, * 10)$ for $\mathrm{S}-1$.
\end{abstract}

Y.-W. Kim and M.-J. Kim contributed equally to this work.

Electronic supplementary material The online version of this article (doi:10.1007/s10120-015-0490-3) contains supplementary material, which is available to authorized users.

Sook Ryun Park

sukryun73@hanmail.net

1 Center for Gastric Cancer, Research Institute and Hospital, National Cancer Center, Goyang, Gyeonggi, Republic of Korea

2 Department of Laboratory Medicine, Center for Diagnostic Oncology, Research Institute and Hospital, National Cancer Center, Goyang, Gyeonggi, Republic of Korea

3 Department of Clinical Pharmacology and Therapeutics, Asan Medical Center, University of Ulsan College of Medicine, Seoul, Republic of Korea

4 Department of Nuclear Medicine, Research Institute and Hospital, National Cancer Center, Goyang, Gyeonggi, Republic of Korea

5 Department of Oncology, Asan Medical Center, University of Ulsan College of Medicine, 88, Olympic-ro, 43-gil, Songpagu, Seoul 138-736, Republic of Korea
Results From October 2006 to June 2008, 44 patients entered the study. 43 eligible patients completed preoperative chemotherapy and 40 completed postoperative chemotherapy. The most common G3/4 toxicities during pre- and postoperative chemotherapy were neutropenia, stomatitis, and abdominal pain. The clinical response rate by RECIST was $74.4 \%$ (95\% CI, 61.4-87.4\%), and the R0 resection rate was $97.7 \%$. Clinical downstaging in T or $\mathrm{N}$ occurred in $41.9 \%$ of patients. The 3-year progressionfree survival (PFS) rate was $62.8 \%$ and 5-year overall survival (OS) rate was $69.6 \%$. PFS and OS differed significantly according to clinical response, clinical downstaging, and CYP2A6 genotype. Patients with CYP2A6 variant/variant genotypes had a higher tegafur $C_{\max }$ and worse survival than those with wild/wild or wild/variant genotypes.

Conclusion Perioperative S-1 plus docetaxel is active with a manageable toxicity in patients with LAGC receiving D2 surgery. Clinical tumor response, clinical downstaging, and CYP2A6 genotype may predict efficacy.

Keywords Gastric cancer - S-1 - Docetaxel · Perioperative chemotherapy $\cdot$ CYP2A6 genotype

\section{Introduction}

Gastric cancer (GC) is a major global health issue and the second leading cause of cancer mortality in the world, with half of all patients in East Asia [1, 2]. In localized GC, survival outcomes after resection differ considerably between Eastern and Western populations, with better survival reported in Eastern studies [3-8]. Although European countries developed perioperative chemotherapy, including neoadjuvant and adjuvant therapy, to overcome poor 
curative resectability and a high risk of relapse, Eastern countries, where the curative resection rate is relatively high and D2 lymph node dissection is routinely performed, developed an adjuvant chemotherapy strategy. Compared to surgery alone, both strategies improve progression-free, disease-free, or relapse-free survival, and overall survival (OS) [3, 4, 7, 9].

In any regions, however, patients with locally advanced GC (LAGC) have a poor prognosis because of the low curative resection rate and the high recurrence rate. In our recently reported randomized phase II trial comparing neoadjuvant versus adjuvant chemotherapy with docetaxel/cisplatin, neoadjuvant chemotherapy was feasible for D2 resected LAGC patients and tended to show downstaged pathological findings and higher $\mathrm{R} 0$ resection rates compared with adjuvant chemotherapy [10]. Only three cycles of chemotherapy in each treatment arm, however, was not likely to overcome a poor prognosis in LAGC.

Given that perioperative chemotherapy has been demonstrated to improve survival outcomes in two randomized controlled trials (MAGIC and FFCD/FNCLCC trials) in the West [7, 9], and postoperative adjuvant chemotherapy after D2 resection has been established as standard therapy based on ACTS-GC and CLASSIC trials in the East $[3,4]$, we combined both preoperative and postoperative approaches to achieve better outcomes in the LAGC patients who have a dismal prognosis.

The current standard of care in advanced GC is fluoropyrimidine plus platinum doublet or epirubicin-containing triplet. However, recently, the platinum-based therapy has been challenged by new-generation agentbased combination regimens because of similar efficacy and better toxicity profiles $[11,12]$. We have also investigated a non-platinum-based regimen, S-1 plus docetaxel, in the phase II study for metastatic GC that showed a high response rate of $66.7 \%$ and good tolerability [13].

Based on these, we performed this phase II study of the combination chemotherapy of $\mathrm{S}-1$ plus docetaxel as perioperative chemotherapy in patients with clinical stage III/ IV (M0) LAGC. In addition, we examined the association between polymorphisms of $C Y P 2 A 6$, a key metabolizer of $\mathrm{S}-1$, and (1) treatment outcomes and (2) pharmacokinetics (PK) of S-1 and its metabolites, and compared PK profiles between pre-gastrectomy and post-gastrectomy.

\section{Methods}

\section{Eligibility}

Eligible patients were 18-70 years old with previously untreated, biopsy-proven LAGC, defined as clinical stage III/IV (M0) disease by the Japanese TNM classification system, which classifies $\mathrm{N}$ stages based on the topographic location of positive nodes rather than their counts [14]. We excluded the patients with clinical stage $\mathrm{N} 3$ by the Japanese system but M1 by the UICC/AJCC TNM system (6th edition) [15]. Clinical $\mathrm{T}$ stage was determined by endoscopic ultrasonography (EUS) and $\mathrm{N}$ stage by abdominal/pelvic computed tomography (CT). Patients underwent ${ }^{18}$ F-fluorodeoxyglucose (FDG)-positron emission tomography (PET) and diagnostic laparoscopy before study entry.

Eligibility also included Eastern Cooperative Oncology Group performance status 0-1, adequate organ function, and measurable lesion(s) by Response Evaluation Criteria in Solid Tumor (RECIST) v. 1.0 [16]. Exclusion criteria included previous malignancy within the past 3 years, except nonmelanomatous skin cancer or carcinoma in situ of the cervix, active infection, or other comorbid conditions that would affect drug tolerance or limit compliance. All patients provided written informed consent, and the Institutional Review Board of the Korean National Cancer Center approved the study.

\section{Treatment}

Preoperative and postoperative chemotherapy consisted of three 3-week cycles of oral S-1 (40 mg/m ${ }^{2}$ twice daily) on days $1-14$ plus intravenous docetaxel $\left(35 \mathrm{mg} / \mathrm{m}^{2}\right)$ on days 1 and 8. Prophylactic granulocyte-colony-stimulating factor was not allowed.

Patients underwent surgery with D2 dissection within 6 weeks of starting preoperative cycle 3 . If disease progressed during preoperative chemotherapy, surgery was performed immediately unless contraindicated. If the tumor was deemed unresectable during or after three cycles of preoperative chemotherapy, the patient did not undergo surgery and was removed from the study. The extent of lymph node dissection was basically D2 or more according to the site of the primary lesion. Postoperative chemotherapy was started 4-6 weeks postoperatively.

\section{Evaluation during the study}

We evaluated toxicity according to the National Cancer Institute Common Terminology Criteria for Adverse Events (v. 3.0). During the first two cycles of preoperative and postoperative chemotherapy, we performed complete blood cell counts with differential and blood chemistry weekly.

We evaluated objective clinical responses after preoperative chemotherapy cycles 2 and 3 using RECIST v 1.0 [16]. We repeated FDG-PET after cycle 1 and EUS after cycle 3 . We defined metabolic response as greater than $35 \%$ decrease of metabolic activity in the primary tumor 
[17]. We used the UICC/AJCC system (6th edition) to determine postoperative pathologic stage [15] and the tumor regression grade system proposed by Dworak et al. [18] to assess pathological response as well as the percentage of vital tumor tissue within the macroscopically identifiable tumor bed (we defined a major response as less than $10 \%$ of residual cancer cells) [19]. R0 resection was defined as resection with no remaining gross or microscopic residual disease.

\section{Genotyping assay and pharmacokinetic study}

We extracted genomic DNA from whole blood and identified the common variant alleles that affect $C Y P 2 A 6$ activity/expression in Asian populations $(C Y P 2 A 6 * 4, * 7, * 9$, and $* 10$ ), along with the wild-type allele $\left(C Y P 2 A 6^{*} 1\right)$, as previously described [20].

For the genotyping assay, we used polymerase chain reaction (PCR)-restriction fragment length polymorphism, sequencing, and primer extension methods to determine three polymorphic sites $(-48 \mathrm{~T}>\mathrm{G}, \quad 6558 \mathrm{~T}>\mathrm{C}$, and $6600 \mathrm{G}>\mathrm{T}$ ) and $C Y P 2 A 6$ deletion.

For the pharmacokinetic study, serial blood samples were collected on day 1 of cycle 1 to characterize the PK of $\mathrm{S}-1$. Blood was drawn before treatment initiation $(0 \mathrm{~h})$, and at $0.5,1,1.5,2,4,6,8$, and $12 \mathrm{~h}$ after treatment initiation on day 1 and pre-dose on day 8 of each first cycle of the preoperative and postoperative treatment. For PK analysis, we determined the plasma concentrations of tegafur, 5-FU, and CDHP, which we measured using liquid chromatography-tandem mass spectrometry (LC/MS/MS). We analyzed PK by non-compartmental methods using WinNonlin 5.2 (Pharsight Corporation, Mountain View, CA, USA). We determined the maximum plasma concentrations $\left(C_{\max }\right)$ and time to $C_{\max }\left(T_{\max }\right)$ directly from the observed values and estimated the terminal elimination rate constant $\left(\lambda_{\mathrm{z}}\right)$ by linear regression of the log-linear decline of at least three individual plasma time-concentration data points. We calculated the terminal half-life $\left(t_{1 / 2}\right)$ for each individual as follows: $t_{1 / 2}=\ln (2) / \lambda_{\mathrm{z}}$. We calculated the area under the plasma concentration-time curve (AUC) from zero to the last measurable time $\left(\mathrm{AUC}_{\text {last }}\right)$ using the linear-log linear trapezoidal method. We also calculated the AUC from time zero extrapolated to infinity $\left(\mathrm{AUC}_{\mathrm{inf}}\right)$ using a combination of the linear-log linear trapezoidal method and an extrapolation to infinity using the $\lambda_{\mathrm{z}}$ and the last observed concentration.

\section{Statistical methods}

The primary endpoint was the clinical response rate of preoperative chemotherapy; the secondary endpoints were progression-free survival (PFS), OS, pathological response, clinical downstaging rate, safety, CYP2A6 genotype, and PK. We calculated sample size using the Simon Minimax two-stage design [21]. Assuming $P_{0}=0.4$ and $P_{1}=0.6$ with $\alpha=0.05$ and $\beta=0.2$, the first stage required a response in more than 18 of 34 patients. If more than 21 of 39 patients responded, further evaluation was warranted. We planned to enroll 44 patients, assuming $10 \%$ would not be evaluable.

We calculated PFS from the start of treatment to the date of recurrence, progression, or death from any cause, and

Table 1 Patient characteristics at baseline

\begin{tabular}{|c|c|c|}
\hline Characteristic & $n$ & $\%$ \\
\hline Eligible patients & 43 & 100 \\
\hline \multicolumn{3}{|l|}{ Sex } \\
\hline Male & 29 & 67.4 \\
\hline Female & 14 & 32.6 \\
\hline \multicolumn{3}{|l|}{ Age, years } \\
\hline Median (range) & $53(33-69)$ & \\
\hline \multicolumn{3}{|l|}{ ECOG performance status } \\
\hline 0 & 2 & 4.7 \\
\hline 1 & 41 & 95.3 \\
\hline \multicolumn{3}{|l|}{ Tumor location in stomach } \\
\hline Upper $1 / 3$ & 9 & 20.9 \\
\hline Lower $2 / 3$ & 26 & 60.5 \\
\hline All over & 8 & 18.6 \\
\hline \multicolumn{3}{|l|}{ Histology } \\
\hline Papillary adenocarcinoma & 1 & 2.3 \\
\hline Adenocarcinoma, well differentiated & 3 & 7.0 \\
\hline Adenocarcinoma, moderately differentiated & 8 & 18.6 \\
\hline Adenocarcinoma, poorly differentiated & 12 & 27.9 \\
\hline Signet-ring cell carcinoma & 16 & 37.2 \\
\hline Mucinous adenocarcinoma & 3 & 7.0 \\
\hline \multicolumn{3}{|l|}{ Lauren classification } \\
\hline Intestinal & 18 & 41.9 \\
\hline Diffuse & 24 & 55.8 \\
\hline Mixed & 1 & 2.3 \\
\hline \multicolumn{3}{|l|}{ Clinical $\mathrm{T}$ classification $^{\mathrm{a}}$} \\
\hline $\mathrm{T} 3$ & 39 & 90.7 \\
\hline $\mathrm{T} 4$ & 4 & 9.3 \\
\hline \multicolumn{3}{|l|}{ Clinical $\mathrm{N}$ classification $^{\mathrm{a}}$} \\
\hline N1 & 21 & 48.8 \\
\hline N2 & 20 & 46.5 \\
\hline N3 & 2 & 4.7 \\
\hline \multicolumn{3}{|l|}{ Clinical TNM stage $^{\mathrm{a}}$} \\
\hline IIIA & 20 & 46.5 \\
\hline IIIB & 18 & 41.9 \\
\hline IV (M0) & 5 & 11.6 \\
\hline
\end{tabular}

a According to the Japanese Classification of Gastric Carcinoma (2nd English edition). T classification was based on EUS, $\mathrm{N}$ classification on CT 
OS to the date of death. We estimated survival using the Kaplan-Meier method and used the log-rank test for comparisons. We used the Pearson chi-squared test and the Fisher exact test to compare discrete data. To compare quantitative data, we used the Mann-Whitney $U$ test for interindividual comparisons and the Wilcoxon signed-rank test for intraindividual comparisons. Logistic regression was used to examine the association between PK parameters and toxicity. $t$ tests and analysis of variance were performed to evaluate the association between polymorphisms and PK parameters. Correlation of the PK between 5-FU and CDHP was examined by linear regression. All tests were two sided, and we considered $P<0.05$ statistically significant. This trial is registered with ClinicalTrials.gov, number NCT00587145.

\section{Results}

\section{Patient characteristics}

Forty-four patients were enrolled from October 2006 to June 2008. One patient with liver metastasis was excluded from the analysis. Median age was 53 years (range, 33-69) with a male:female ratio of 2.1:1. More than half had clinical IIIB/IV (M0) disease according to the JCGC staging system (Table 1).

\section{Chemotherapy delivery and toxicity}

All 43 eligible patients completed the three preoperative chemotherapy cycles and $40(93.0 \%)$ also completed the three postoperative chemotherapy cycles. For 3 patients, the reasons for not starting postoperative chemotherapy were peritoneal seeding found at laparotomy $(n=1)$ and no pathological regression $(n=2)$.

Table 2 summarizes adverse events per patient. The most common grade 3/4 toxicity during pre- and postoperative chemotherapy was neutropenia $(27.9 \%$ vs. $65.0 \%$ ), and grade 3 febrile neutropenia occurred in 3 and 2 patients, respectively ( $7.0 \%$ vs. $5.0 \%$ ). The most common grade 3 nonhematological toxicities during pre- and postoperative chemotherapy were stomatitis $(18.6 \%$ vs. $5.0 \%)$ and abdominal pain $(4.7 \%$ vs. $17.5 \%)$, respectively. Preoperative chemotherapy was better tolerated, with lower incidences of grade 3/4 leukopenia $(P=0.006)$, neutropenia $(P=0.001)$, abdominal pain $(P=0.081)$, and overall grade $3 / 4$ toxicity $(48.8 \%$ vs. $72.5 \% ; \quad P=0.043), \quad$ compared with postoperative chemotherapy.

A total of 249 cycles were administered, and dose reduction of S-1 and/or docetaxel was required in 63 $(25.3 \%)$ cycles in 16 patients (37.2\%), primarily because of grade 3 stomatitis (6 patients), grade 3 febrile neutropenia (5 patients), and grade 3 abdominal pain (4

Table 2 Adverse events during preoperative and postoperative chemotherapy

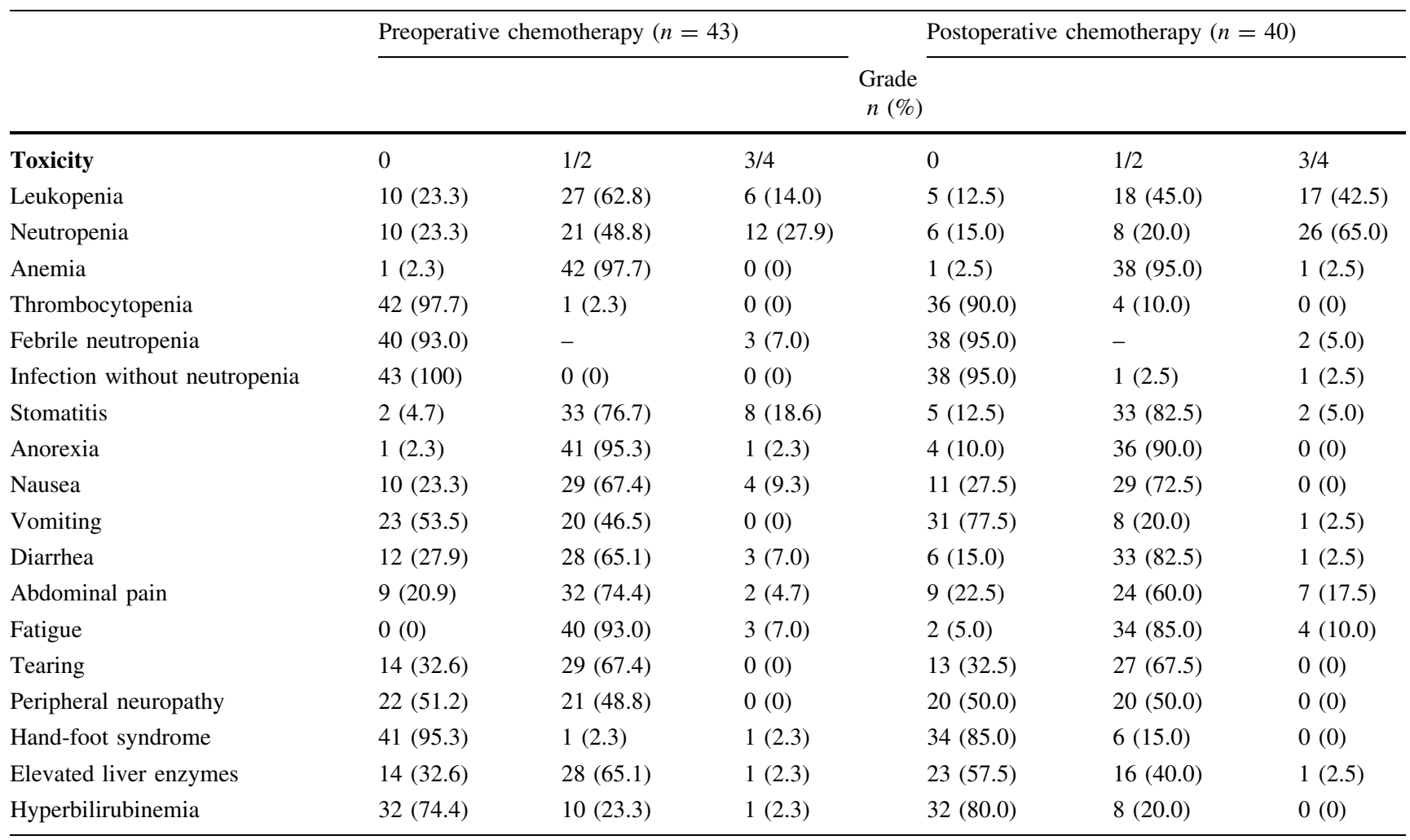


patients). The relative dose intensity of docetaxel was $97.1 \%$ preoperatively and $90.6 \%$ postoperatively, and that of S-1 was $95.2 \%$ preoperatively and $87.5 \%$ postoperatively.

\section{Pharmacokinetics and pharmacogenetics}

PK analysis of tegafur, 5-fluorouracil (5-FU), and 5-chloro2,4-dihydroxypyridine (CDHP) was performed in 37 patients (Online Resource 1). The comparison between preoperative and postoperative PK profiles of S-1 was reported elsewhere, and we here report the preoperative PK results, normalized at $70 \mathrm{mg}$ of S-1. S-1 was absorbed rapidly with median $T_{\max }$ of tegafur of $1.02 \mathrm{~h}$ after treatment. Plasma concentrations varied widely, and the $\mathrm{AUC}_{\mathrm{inf}}$ of 5-FU correlated with the $\mathrm{AUC}_{\text {inf }}$ of CDHP, which suggested the inhibitory effect of CDHP on the degradation of 5-FU by DPD $\left(P=0.054, R^{2}=0.11\right)$ (Fig. 1).

$C Y P 2 A 6$ genotyping was performed in all 43 eligible patients, and the observed allelic frequencies were 0.58 for $C Y P 2 A 6^{*} 1,0.12$ for $C Y P 2 A 6^{*} 4,0.03$ for $C Y P 2 A 6^{*} 7,0.23$ for $C Y P 2 A 6^{*} 9$, and 0.03 for $C Y P 2 A 6^{*} 10$, which were similar to the frequencies previously reported in Asian populations $[22,23]$. When the variants were combined into a variant genotype $(V)$, the frequencies of genotypes were as follows: $W / W(n=13,30.2 \%), W / V(n=24$, $55.8 \%)$, and $V / V(n=6,13.95 \%)$. In 37 patients who had both PK and PG data, patients with 0 or 1 variant allele, wild/wild $(W W)$ or wild/variant $(W / V)$, had a lower mean $C_{\max }(20.3 \pm 1.2$ vs. $27.7 \pm 4.6 ; P=0.045)$ and $\mathrm{AUC}_{0 \text {-inf }}$ $(172.5 \pm 12.5$ vs. $220.4 \pm 43.1 ; P=0.187)$ for tegafur than those with two variant alleles $(V / V)$, whereas patients with $W / W$ or $W / V$ had a higher $C_{\max }(1.8 \pm 0.1$ vs. $1.4 \pm 0.2 ; \quad P=0.178)$ and $\mathrm{AUC}_{0 \text {-inf }}(9.7 \pm 0.5$ vs. $8.4 \pm 1.2 ; P=0.308)$ for 5 -FU than those with $V / V$. The ratio between exposure to 5 -FU and tegafur $\left(\mathrm{AUC}_{0 \text {-inf }}\right.$ of 5 -FU/AUC ${ }_{0 \text {-inf }}$ of tegafur) was $0.06594 \pm 0.005422$ in patients with $W / W$ or $W / V$ genotypes and $0.04385 \pm$ 0.008376 in patients with $V / V(P=0.1292)$ (Fig. 2). Neither genotype nor PK was significantly associated with toxicities (Online Resource 2).

\section{Clinical and metabolic responses to preoperative chemotherapy}

One of 43 patients $(2.3 \%)$ had a complete response (CR) to preoperative chemotherapy, $31(72.1 \%)$ had a partial response (PR), 9 (20.9\%) had a stable disease (SD), and 2 $(4.7 \%)$ had a progressive disease (PD). The overall response rate was $74.4 \%$ (95\% confidence interval, 61.4-87.4\%). Clinical T downstaging based on EUS occurred in 7 patients $(16.3 \%), \mathrm{N}$ downstaging based on CT developed in $16(37.2 \%)$, with clinical downstaging for
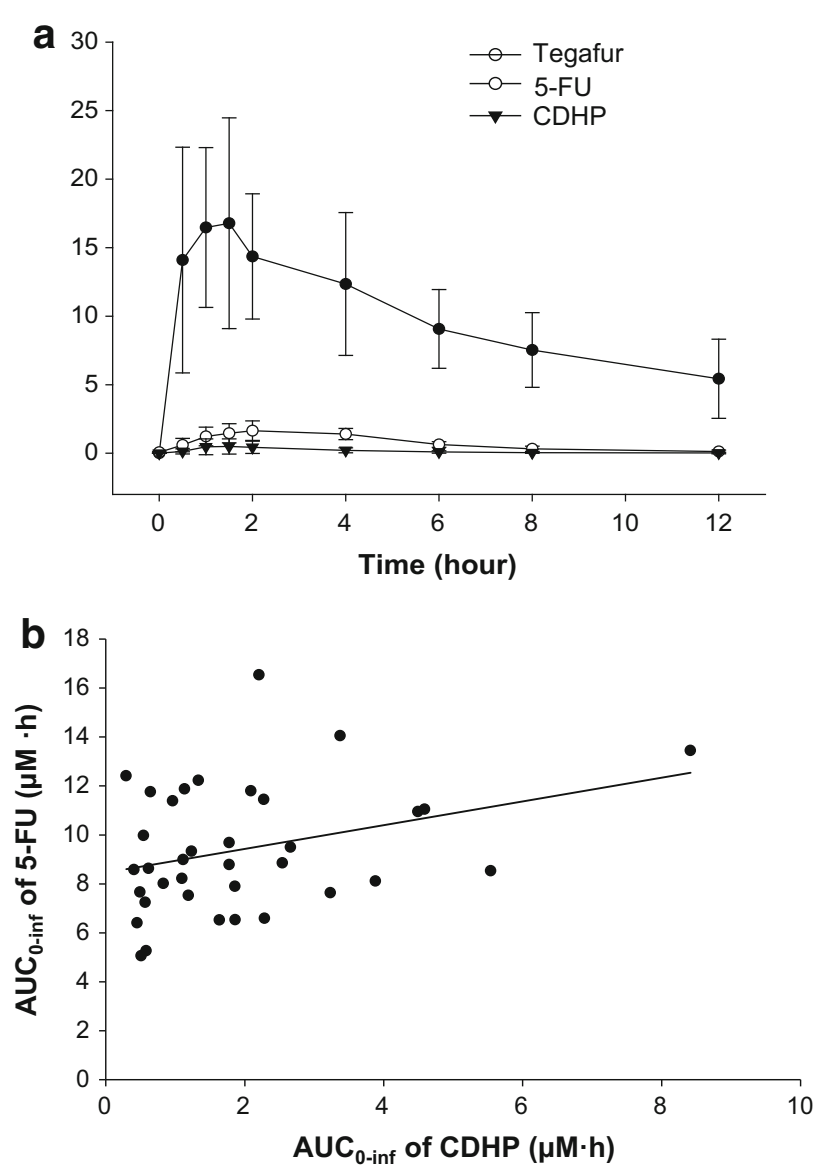

Fig. 1 a Plasma concentrations of tegafur, 5-fluorouracil (5-FU), and 5-chloro-2,4-dihydroxypyridine (CDHP) after S-1 administration, normalized to $70 \mathrm{mg}$ before gastrectomy. b Correlation between $\mathrm{AUC}_{0 \text {-inf }}$ of 5 -FU and CDHP

either $\mathrm{T}$ or $\mathrm{N}$, in $18(41.9 \%)$. The baseline FDG SUV $\max$ in the 42 patients who had FDG-avid primary tumors was $7.03 \pm 5.11$ (mean \pm SD). Mean FDG uptake significantly decreased to $4.75 \pm 2.55$ after chemotherapy, with a median change of $-26.0 \%(P<0.001) .13$ patients $(31.0 \%)$ had a metabolic response.

\section{Surgical outcomes, pathological stage, and pathological response}

Forty-two patients $(97.7 \%$ ) underwent curative R0 resection with D2 dissection; 1 patient did not undergo resection because unsuspected peritoneal carcinomatosis was discovered at surgery; 24 patients $(57.1 \%)$ underwent total gastrectomy, and the median number of dissected lymph nodes was 46 (range, 24-96). The proportion of stages was $79.1 \%$ for pT0-2, $46.5 \%$ for pN0, and $67.4 \%$ for stage 0 -II. Ten patients $(23.3 \%)$ showed grade $\geq 3$ tumor regression [very few (difficult to find microscopically) tumor cells in fibrotic tissue], which included one complete regression, and 22 $(51.2 \%)$ showed a pathological major response (Table 3). 
Fig. 2 Association between pharmacokinetic profile and $C Y P 2 A 6$ genotype according to CYP2A6 genotype: $C_{\max }(\mathbf{a})$ and $\mathrm{AUC}_{0 \text {-inf }}(\mathbf{b})$ of tegafur, $C_{\max }$ (c) and $\mathrm{AUC}_{0 \text {-inf }}(\mathbf{d})$ of 5-fluorouracil (5-FU), and ratio of the $\mathrm{AUC}_{0 \text {-inf }}$ of 5 -FU to the $\mathrm{AUC}_{0 \text {-inf }}$ of tegafur (e). $W$ wildtype allele, $V$ variant allele
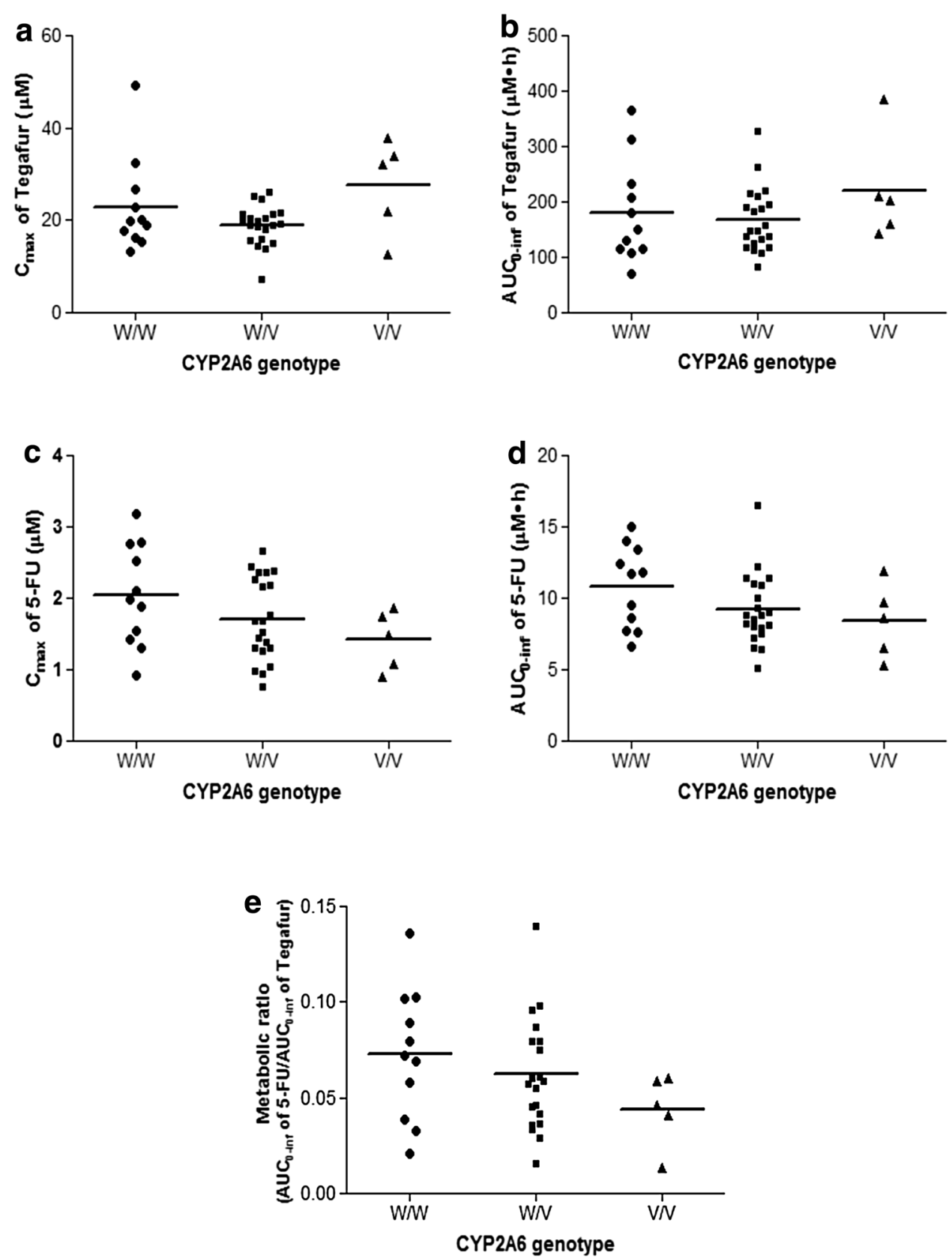

Postoperative complications occurred in 18 patients $(42.9 \%)$ and included two major cases of intraabdominal abscesses and bleeding at anastomosis site, but there was no 30-day postoperative mortality. Other complications included fever $(n=13)$, atelectasis $(n=7)$, ileus $(n=3)$, delayed wound healing $(n=1)$, pneumonia $(n=1)$, and anastomosis site stricture $(n=1)$. The median hospital stay after surgery was 10 days (range, 8-17 days).

\section{Overall survival and progression-free survival}

With a median follow-up of 66.6 months (range, 53.7-75.5), the median OS and PFS were not reached. The mean 3-year PFS rate was $62.8 \% \pm 7.4 \%$ and the 5-year OS rate was $69.6 \% \pm 7.0 \%$. Both rates differed significantly according to the clinical response to preoperative chemotherapy. The 3-year PFS rate was $78.1 \% \pm 7.3 \%$ in patients with CR/PR, $22.2 \% \pm 13.9 \%$ in patients with $\mathrm{SD}$, and $0 \%$ in patients with $\mathrm{PD}(P<0.0001)$. The 5-year OS rate was $81.1 \% \pm 6.9 \%$ in patients with CR/PR, $44.4 \% \pm 16.6 \%$ in patients with $\mathrm{SD}$, and $0 \%$ in patients with PD $(P<0.001)$ (Fig. 3a, b). Clinical downstaging in $\mathrm{T}$ and/or $\mathrm{N}$ was also associated with survival [83.3 \% $\pm 8.8 \%$ vs. $48.0 \% \pm 10.0 \%$ for 3-year PFS rate $(P=0.013)$ and $83.0 \% \pm 9.0 \%$ vs. $60.0 \% \pm 9.8 \%$ for 5-year OS rate $(P=0.055)$ ] (Fig. 3c, d). Notably, survival 
Table 3 Pathological stage and response

\begin{tabular}{|c|c|c|}
\hline Characteristic & $n$ & $\%$ \\
\hline Patients eligible & 43 & 100 \\
\hline Patients undergoing resection & 42 & 97.7 \\
\hline \multicolumn{3}{|l|}{$\mathrm{T}$ classification } \\
\hline T0 & 1 & 2.3 \\
\hline $\mathrm{T} 1$ & 5 & 11.6 \\
\hline $\mathrm{T} 2$ & 28 & 65.1 \\
\hline $\mathrm{T} 3$ & 5 & 11.6 \\
\hline $\mathrm{T} 4$ & 3 & 7.0 \\
\hline Not evaluable $^{\mathrm{a}}$ & 1 & 2.3 \\
\hline \multicolumn{3}{|l|}{$\mathrm{N}$ classification (nodes involved) } \\
\hline N0 (0) & 20 & 46.5 \\
\hline N1 $(<7)$ & 12 & 27.9 \\
\hline N2 (7-14) & 8 & 18.6 \\
\hline N3 $(>14)$ & 2 & 4.7 \\
\hline Not evaluable ${ }^{a}$ & 1 & 2.3 \\
\hline \multicolumn{3}{|l|}{ TNM stage } \\
\hline 0 & 1 & 2.3 \\
\hline IA & 2 & 4.7 \\
\hline IB & 17 & 39.5 \\
\hline II & 9 & 20.9 \\
\hline IIIA & 8 & 18.6 \\
\hline IIIB & 1 & 2.3 \\
\hline IV (M0) & 4 & 9.3 \\
\hline IV $(\mathrm{M} 1)^{\mathrm{a}}$ & 1 & 2.3 \\
\hline \multicolumn{3}{|l|}{ Pathological response } \\
\hline \multicolumn{3}{|l|}{ Dworak grade } \\
\hline 0 & 2 & 4.7 \\
\hline 1 & 10 & 23.3 \\
\hline 2 & 20 & 46.5 \\
\hline 3 & 9 & 20.9 \\
\hline 4 & 1 & 2.3 \\
\hline Not evaluable $^{\mathrm{a}}$ & 1 & 2.3 \\
\hline
\end{tabular}

Pathological stage was based on the 6th edition of the UICC/AJCC staging system

${ }^{a}$ Patient did not undergo resection because of distant metastases and was assigned to stage IV (M1)

differed according to CYP2A6 genotype. The 3-year PFS rate was $67.6 \% \pm 7.7 \%$ in patients with $W / W$ or $W / V$ and $33.3 \% \pm 19.2 \%$ in patients with $V / V \quad(P=0.102)$, whereas the 5-year OS rate was $75.6 \% \pm 7.1 \%$ in patients with $W / W$ or $W / V$ and $33.3 \% \pm 19.2 \%$ in patients with $V / V(P=0.032)$ (Fig. 3e, f).

Regarding pathological response, neither achievement of major response nor a higher grade of tumor regression was associated with a better prognosis. Patients with no evidence of pathological regression (grade 0), however, had extremely poor PFS compared with those with any grade of regression (median PFS, 6.4 months vs. not reached, $P<0.001$ ) (Fig. 4a, b). Metabolic response was also not significantly associated with survival (Fig. 4c, d).

\section{Discussion}

We demonstrated here that perioperative S-1 plus weekly docetaxel was highly efficacious and well tolerated in patients with LAGC. Clinical response rate was $74.4 \%$, and clinical downstaging occurred in $41.9 \%$. The R0 resection rate was $97.7 \%$ and major pathological response rate was $51.2 \%$. The 3-year PFS rate was $62.8 \%$ and 5-year OS rate was $69.6 \%$. With a lack of validated surrogate endpoints for survival in the neoadjuvant setting for GC, R0 resection has been shown to be the most consistent prognostic indicator [24]. Although staging laparoscopy might have partly contributed to our results, the R0 resection rate $(97.7 \%)$ was very high given that the R0 rate was $76.1 \%$ in clinical stage III and $55.1 \%$ in stage IV (M0) GC (without neoadjuvant therapy) in our prospective database [5] and $81.9 \%-90.9 \%$ in previous neoadjuvant studies where patients had relatively less advanced GC and staging laparoscopy was also performed [8, 24, 25].

As the present trial was only a small phase II trial with objective tumor response rate as a primary endpoint, it is difficult to compare directly the outcomes of our study and previous randomized phase III trials of perioperative chemotherapy [7, 9]. However, given that the present study was performed in patients with locally advanced disease, which was defined as clinical stage III/IV (M0) whereas MAGIC and FFCD/FNCLCC trials enrolled patients with resectable GC with stage II or higher, our results seem to be promising compared to those achieved with platinumbased perioperative chemotherapy of ECF (MAGIC) or CF (FFCD/FNCLCC) in terms of R0 resection rate $(69.3 \%$ in MAGIC and $84 \%$ in FFCD/FNCLCC) and 5-year OS rate (36\% in MAGIC and $38 \%$ in FFCD/FNCLCC). Meanwhile, one of the criticisms of the MAGIC and FFCD/ FNCLCC trials was that more than half of the patients were not able to complete planned perioperative chemotherapy. In contrast, $93 \%$ of patients completed all six cycles of perioperative chemotherapy in our study, and the dose intensity of chemotherapy was well maintained (relative dose intensity: docetaxel with 0.97 preoperatively and 0.91 postoperatively, and S-1 with 0.95 and 0.88 , respectively). These results suggest that the non-platinum-based combination of S-1 plus docetaxel has excellent feasibility and tolerability in the perioperative chemotherapy setting.

S-1-containing platinum-based doublet or triplet regimens have been also evaluated in the neoadjuvant setting, especially in East Asia [26, 27]. Neoadjuvant S-1 plus cisplatin resulted in a promising $\mathrm{R} 0$ resection rate of $82 \%$ in 53 LAGC patients with extensive lymph node metastasis 

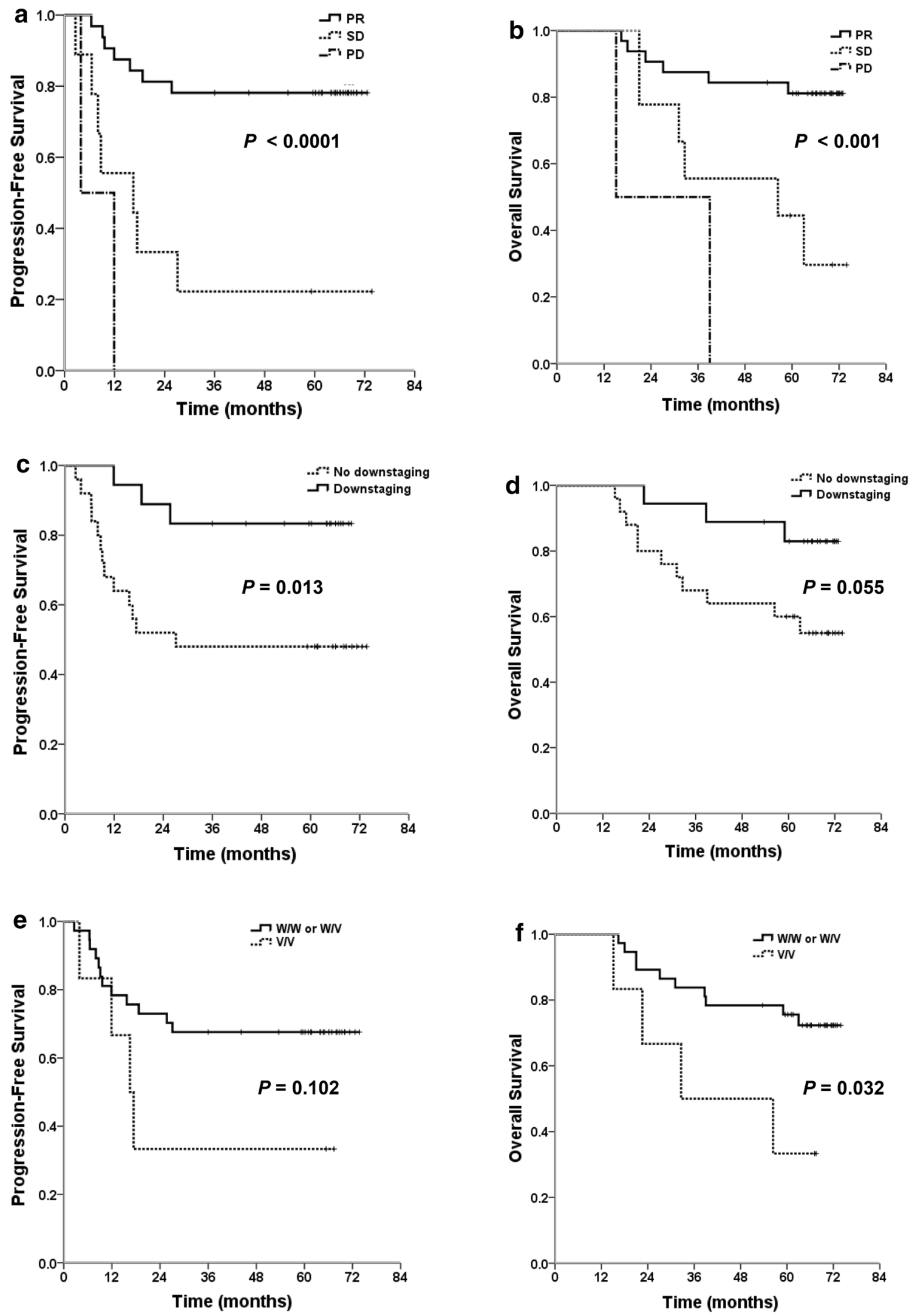

Fig. 3 Kaplan-Meier curves of progression-free survival (PFS) (a) and overall survival (OS) (b) according to clinical response, PFS (c) and OS (d) according to clinical downstaging, and PFS (e) and OS (f) according to CYP2A6 genotype. $W$ wild-type allele, $V$ variant allele 

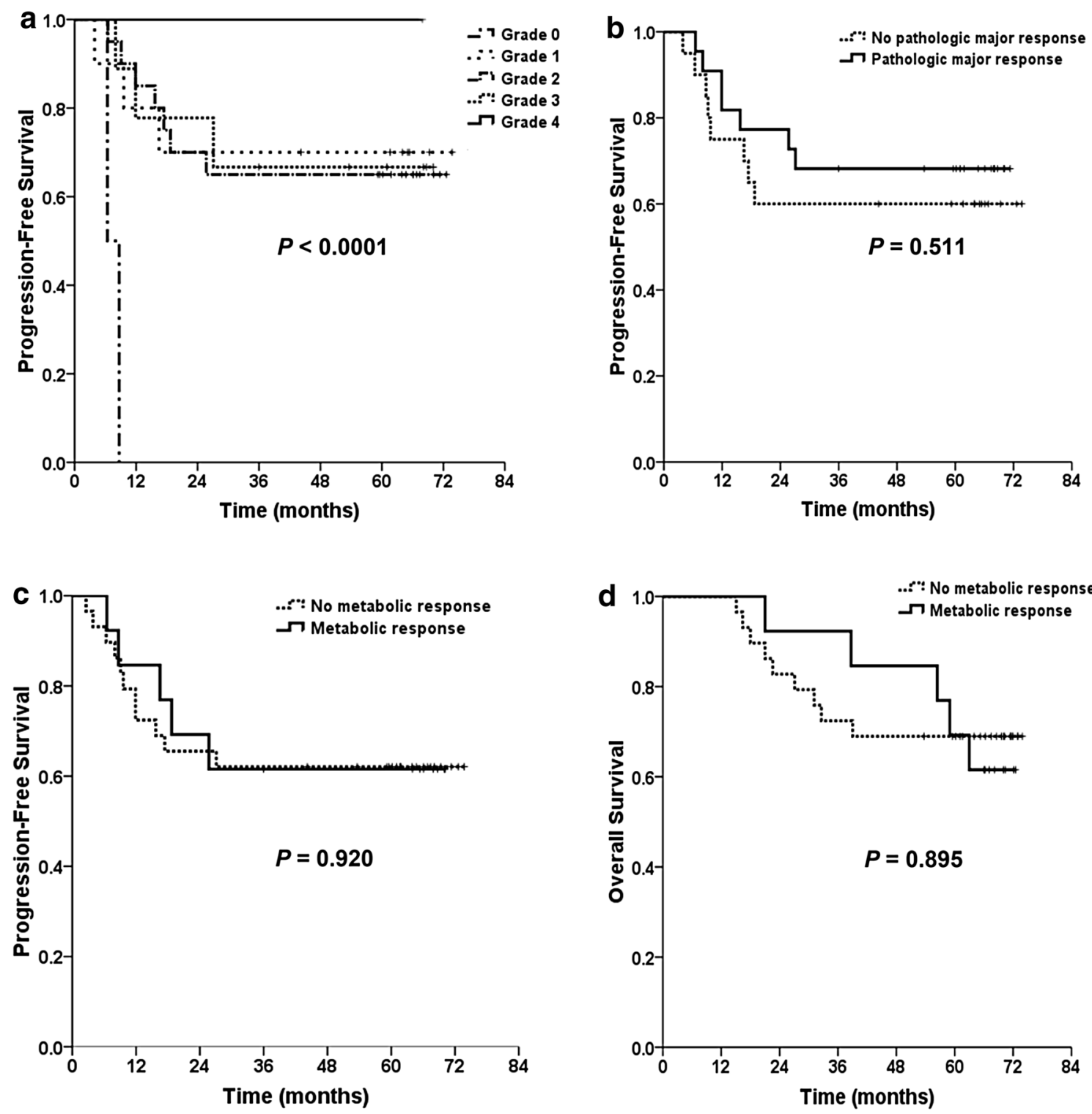

Fig. 4 Kaplan-Meier curves of progression-free survival (PFS) according to pathological response by Dworak criteria (a), PFS according to major pathological response (residual cancer cells $<10 \%$ ) (b), PFS (c) and overall survival (OS) (d) according to

[26]. Although the relatively low dose of cisplatin $(60 \mathrm{mg} /$ $\mathrm{m}^{2}$ every 4 weeks, $15 \mathrm{mg} / \mathrm{m}^{2} /$ week) in this study might contribute to a favorable toxicity profile, cisplatin still requires intensive intravenous hydration to prevent nephrotoxicity. Neoadjuvant docetaxel, oxaliplatin, and S-1 (DOS) also resulted in a high R0 resection rate of $97.6 \%$ in 41 patients with potentially resectable GC [27]. Although the triplet regimen resulted in relatively higher toxicities (grade $\geq 3$ neutropenia $65.6 \%$, grade $\geq 3$ thrombocytopenia $17.1 \%$, neutropenic fever $9.8 \%$ ), based on high activity, DOS regimen was selected as preoperative therapy in the ongoing phase III PRODIGY study (NCT01515748). metabolic response, defined as a decrease $>35 \%$ of metabolic activity in the primary tumor on FDG-PET performed after the first preoperative cycle

Further studies are warranted to determine better chemotherapy among doublet versus triplet or platinum-based versus non-platinum-based regimens in the neoadjuvant setting.

Recently, Oki et al. evaluated the efficacy and safety of neoadjuvant docetaxel and S-1 combination in stage III resectable GC [28]. The clinical response rate using RECIST v 1.0 was $34 \%$, which was lower than that in our study $(74 \%)$. One possible explanation might be the lower planned dose intensities in both docetaxel and S-1 in that study in which docetaxel $35 \mathrm{mg} / \mathrm{m}^{2}$ was administered on days 1 and 15 and S-1 $40 \mathrm{mg} / \mathrm{m}^{2}$ was given twice daily for 
days 1-14 every 4 weeks for 2 cycles. Planned dose intensity was $17.5 \mathrm{mg} / \mathrm{m}^{2} /$ week for docetaxel and $280 \mathrm{mg} /$ $\mathrm{m}^{2} /$ week for $\mathrm{S}-1$ in that study, whereas in our study, it was $23.3 \mathrm{mg} / \mathrm{m}^{2} /$ week and $373.3 \mathrm{mg} / \mathrm{m}^{2} /$ week, respectively. In addition, 7 of 47 patients (15\%) in their study did not complete neoadjuvant chemotherapy because of adverse events. A recent phase III trial with first-line S-1 plus docetaxel in advanced GC, in which both agents were also administered at lower doses compared to our regimen, reported a response rate of $38.8 \%$, which was lower than $66.7 \%$ in our previous study for metastatic GC [13, 29].

Our finding of an association between clinical response by RECIST and long-term survival was consistent with previous studies [24, 30], as was our finding that clinical downstaging was also significantly associated with better survival, consistent with our previous report [31]. These results suggest that reduction in tumor size by RECIST or downstaging might reflect a reduction of tumor burden and may be a useful clinical parameter with which to predict better survival outcome in LAGC.

Furthermore, our results showing an association between CYP2A6 genotype and survival following S-1/docetaxel chemotherapy are consistent with our previous results in metastatic $\mathrm{GC}$, in which the CYP2A6 genotype correlated with the efficacy of S-1-based chemotherapy [20,32]. This finding is consistent with the PK differences associated with CYP $2 A 6$ genotype as shown in the present and previous studies $[33,34]$ and suggests that patients with a $V / V$ genotype might not be good candidates for S-1-based chemotherapy.

Whether pathological response is an indicator of efficacy for neoadjuvant treatment for GC is controversial [35-37]. In this study, pathological response did not correlate with survival, but grade 0 (no evidence of tumor regression) was associated with extremely poor survival. Further prospective studies using standardized criteria are needed to clarify the prognostic role of a pathological response. Similarly, the prognostic value of metabolic response by FDG-PET in GC is also controversial. Although one study reported that a decrease of more than $35 \%$ in FDG uptake after 2 weeks of chemotherapy correlated with OS [38, 39], other studies showed no such correlation [40, 41]. The inconsistent results may have been caused, in part, by differences in the criteria used for metabolic response and in the time intervals between chemotherapy and evaluation.

There are some limitations because this is a small phase II study performed in a single institution with limited statistical power. A future randomized study in a larger cohort is warranted to confirm our findings.

In conclusion, perioperative $\mathrm{S}-1$ plus docetaxel is an active regimen with a manageable toxicity profile in patients with LAGC undergoing D2 surgery. Clinical tumor response, clinical downstaging, and $C Y P 2 A 6$ genotypes may be predictive of efficacy. Based on the promising results observed in our study, the combination of S-1 plus docetaxel could be evaluated in a future phase III study in comparison with the established regimens, ECF and $\mathrm{CF}$, in terms of efficacy and safety in the perioperative setting.

Acknowledgments This work was supported by the Research Institute and Hospital, National Cancer Center, Republic of Korea (Grant 0610520). S-1 was provided by JEIL Pharmaceutical Co., Ltd., and docetaxel by sanofi-aventis Korea Co., Ltd. We thank Min Kyeong Kim for technical assistance.

\section{References}

1. Jemal A, Bray F, Center MM, Ferlay J, Ward E, Forman D. Global cancer statistics. CA Cancer J Clin. 2011;61:69-90.

2. Jung KW, Won YJ, Kong HJ, Oh CM, Seo HG, Lee JS. Cancer statistics in Korea: incidence, mortality, survival and prevalence in 2010. Cancer Res Treat. 2013;45:1-14.

3. Sakuramoto S, Sasako M, Yamaguchi T, Kinoshita T, Fujii M, Nashimoto A, et al. Adjuvant chemotherapy for gastric cancer with S-1, an oral fluoropyrimidine. N Engl J Med. 2007;357:1810-20.

4. Bang YJ, Kim YW, Yang HK, Chung HC, Park YK, Lee KH, et al. Adjuvant capecitabine and oxaliplatin for gastric cancer after D2 gastrectomy (CLASSIC): a phase 3 open-label, randomised controlled trial. Lancet. 2012;379:315-21.

5. Park SR, Kim MJ, Ryu KW, Lee JH, Lee JS, Nam BH, et al. Prognostic value of preoperative clinical staging assessed by computed tomography in resectable gastric cancer patients: a viewpoint in the era of preoperative treatment. Ann Surg. 2010;251:428-35.

6. Macdonald JS, Smalley SR, Benedetti J, Hundahl SA, Estes NC, Stemmermann GN, et al. Chemoradiotherapy after surgery compared with surgery alone for adenocarcinoma of the stomach or gastroesophageal junction. N Engl J Med. 2001;345:725-30.

7. Cunningham D, Allum WH, Stenning SP, Thompson JN, Van de Velde CJ, Nicolson M, et al. Perioperative chemotherapy versus surgery alone for resectable gastroesophageal cancer. N Engl J Med. 2006;355:11-20.

8. Schuhmacher C, Gretschel S, Lordick F, Reichardt P, Hohenberger W, Eisenberger $\mathrm{CF}$, et al. Neoadjuvant chemotherapy compared with surgery alone for locally advanced cancer of the stomach and cardia: European Organisation for Research and Treatment of Cancer randomized trial 40954. J Clin Oncol. 2010;28:5210-8.

9. Ychou M, Boige V, Pignon JP, Conroy T, Bouche O, Lebreton G, et al. Perioperative chemotherapy compared with surgery alone for resectable gastroesophageal adenocarcinoma: an FNCLCC and FFCD multicenter phase III trial. J Clin Oncol. 2011;29:1715-21.

10. Chun J, Park S, Kim H, Kim Y, Ryu K, Lee J, et al. Randomized phase II trial of neoadjuvant vs. adjuvant docetaxel plus cisplatin in patients with locally advanced gastric carcinoma: an interim analysis. J Clin Oncol. 2006;24:18S (abstract \#4030).

11. Dank M, Zaluski J, Barone C, Valvere V, Yalcin S, Peschel C, et al. Randomized phase III study comparing irinotecan combined with 5-fluorouracil and folinic acid to cisplatin combined with 5 -fluorouracil in chemotherapy naive patients with advanced adenocarcinoma of the stomach or esophagogastric junction. Ann Oncol. 2008;19:1450-7. 
12. Jeung HC, Rha SY, Im CK, Shin SJ, Ahn JB, Yang WI, et al. A randomized phase 2 study of docetaxel and S-1 versus docetaxel and cisplatin in advanced gastric cancer with an evaluation of SPARC expression for personalized therapy. Cancer. 2011;117:2050-7.

13. Park SR, Kim HK, Kim CG, Choi IJ, Lee JS, Lee JH, et al. Phase I/II study of S-1 combined with weekly docetaxel in patients with metastatic gastric carcinoma. Br J Cancer. 2008;98:1305-11.

14. Japanese Gastric Cancer. A. Japanese classification of gastric carcinoma, 2nd English edition. Gastric Cancer. 1998;1:10-24.

15. Sobin LH, Wittekind C. TNM classification of malignant tumors. 6th ed. New York: Wiley-Liss; 2002.

16. Therasse P, Arbuck SG, Eisenhauer EA, Wanders J, Kaplan RS, Rubinstein $\mathrm{L}$, et al. New guidelines to evaluate the response to treatment in solid tumors. European Organization for Research and Treatment of Cancer, National Cancer Institute of the United States, National Cancer Institute of Canada. J Natl Cancer Inst. 2000;92:205-16.

17. Ott K, Weber WA, Lordick F, Becker K, Busch R, Herrmann K, et al. Metabolic imaging predicts response, survival, and recurrence in adenocarcinomas of the esophagogastric junction. J Clin Oncol. 2006;24:4692-8.

18. Dworak O, Keilholz L, Hoffmann A. Pathological features of rectal cancer after preoperative radiochemotherapy. Int $\mathrm{J}$ Colorectal Dis. 1997;12:19-23.

19. Ajani JA, Mansfield PF, Lynch PM, Pisters PW, Feig B, Dumas $\mathrm{P}$, et al. Enhanced staging and all chemotherapy preoperatively in patients with potentially resectable gastric carcinoma. J Clin Oncol. 1999;17:2403-11.

20. Kong SY, Lim HS, Nam BH, Kook MC, Kim YW, Ryu KW, et al. Association of CYP2A6 polymorphisms with S-1 plus docetaxel therapy outcomes in metastatic gastric cancer. Pharmacogenomics. 2009;10:1147-55.

21. Simon R. Optimal two-stage designs for phase II clinical trials. Control Clin Trials. 1989;10:1-10.

22. Kwon JT, Nakajima M, Chai S, Yom YK, Kim HK, Yamazaki H, et al. Nicotine metabolism and CYP2A6 allele frequencies in Koreans. Pharmacogenetics. 2001;11:317-23.

23. Nakajima M, Fukami T, Yamanaka H, Higashi E, Sakai H, Yoshida R, et al. Comprehensive evaluation of variability in nicotine metabolism and CYP2A6 polymorphic alleles in four ethnic populations. Clin Pharmacol Ther. 2006;80:282-97.

24. Schuhmacher CP, Fink U, Becker K, Busch R, Dittler HJ, Mueller $\mathrm{J}$, et al. Neoadjuvant therapy for patients with locally advanced gastric carcinoma with etoposide, doxorubicin, and cisplatinum. Closing results after 5 years of follow-up. Cancer (Phila). 2001;91:918-27.

25. Li ZY, Koh CE, Bu ZD, Wu AW, Zhang LH, Wu XJ, et al. Neoadjuvant chemotherapy with FOLFOX: improved outcomes in Chinese patients with locally advanced gastric cancer. J Surg Oncol. 2012;105:793-9.

26. Tsuburaya A, Mizusawa J, Tanaka Y, Fukushima N, Nashimoto A, Sasako M, et al. Neoadjuvant chemotherapy with S-1 and cisplatin followed by D2 gastrectomy with paraaortic lymph node dissection for gastric cancer with extensive lymph node metastasis. Br J Surg. 2014;101:653-60.

27. Park I, Ryu MH, Choi YH, Kang HJ, Yook JH, Park YS, et al. A phase II study of neoadjuvant docetaxel, oxaliplatin, and S-1 (DOS) chemotherapy followed by surgery and adjuvant S-1 chemotherapy in potentially resectable gastric or gastroesophageal junction adenocarcinoma. Cancer Chemother Pharmacol. 2013;72:815-23.
28. Oki E, Emi Y, Kusumoto T, Sakaguchi Y, Yamamoto M, Sadanaga N, et al. Phase II study of docetaxel and S-1 (DS) as neoadjuvant chemotherapy for clinical stage III resectable gastric cancer. Ann Surg Oncol. 2014;21:2340-6.

29. Koizumi W, Kim YH, Fujii M, Kim HK, Imamura H, Lee KH, et al. Addition of docetaxel to $\mathrm{S}-1$ without platinum prolongs survival of patients with advanced gastric cancer: a randomized study (START). J Cancer Res Clin Oncol. 2014;140:319-28.

30. Liu K, Li G, Fan C, Zhou C, Li J. Adapted Choi response criteria for prediction of clinical outcome in locally advanced gastric cancer patients following preoperative chemotherapy. Acta Radiol. 2012;53:127-34.

31. Park SR, Lee JS, Kim CG, Kim HK, Kook MC, Kim YW, et al. Endoscopic ultrasound and computed tomography in restaging and predicting prognosis after neoadjuvant chemotherapy in patients with locally advanced gastric cancer. Cancer. 2008;112:2368-76.

32. Park SR, Kong SY, Nam BH, Choi IJ, Kim CG, Lee JY, et al. CYP2A6 and ERCC1 polymorphisms correlate with efficacy of $\mathrm{S}-1$ plus cisplatin in metastatic gastric cancer patients. $\mathrm{Br} \mathrm{J}$ Cancer. 2011;104:1126-34.

33. Fujita K, Yamamoto W, Endo S, Endo H, Nagashima F, Ichikawa $\mathrm{W}$, et al. CYP2A6 and the plasma level of 5-chloro-2,4-dihydroxypyridine are determinants of the pharmacokinetic variability of tegafur and 5-fluorouracil, respectively, in Japanese patients with cancer given S-1. Cancer Sci. 2008;99:1049-54.

34. Kaida Y, Inui N, Suda T, Nakamura H, Watanabe H, Chida K. The CYP2A6*4 allele is determinant of S-1 pharmacokinetics in Japanese patients with non-small-cell lung cancer. Clin Pharmacol Ther. 2008;83:589-94.

35. Becker K, Reim D, Novotny A, Zum Buschenfelde CM, Engel J, Friess $\mathrm{H}$, et al. Proposal for a multifactorial prognostic score that accurately classifies 3 groups of gastric carcinoma patients with different outcomes after neoadjuvant chemotherapy and surgery. Ann Surg. 2012;256:1002-7.

36. Mansour JC, Tang L, Shah M, Bentrem D, Klimstra DS, Gonen $\mathrm{M}$, et al. Does graded histologic response after neoadjuvant chemotherapy predict survival for completely resected gastric cancer? Ann Surg Oncol. 2007;14:3412-8.

37. Ajani JA, Mansfield PF, Crane CH, Wu TT, Lunagomez S, Lynch PM, et al. Paclitaxel-based chemoradiotherapy in localized gastric carcinoma: degree of pathologic response and not clinical parameters dictated patient outcome. J Clin Oncol. 2005;23:1237-44.

38. Ott K, Fink U, Becker K, Stahl A, Dittler HJ, Busch R, et al. Prediction of response to preoperative chemotherapy in gastric carcinoma by metabolic imaging: results of a prospective trial. J Clin Oncol. 2003;21:4604-10.

39. Ott K, Herrmann K, Lordick F, Wieder H, Weber WA, Becker K, et al. Early metabolic response evaluation by fluorine-18 fluorodeoxyglucose positron emission tomography allows in vivo testing of chemosensitivity in gastric cancer: long-term results of a prospective study. Clin Cancer Res. 2008;14:2012-8.

40. Vallbohmer D, Holscher AH, Schneider PM, Schmidt M, Dietlein $\mathrm{M}$, Bollschweiler E, et al. $\left[{ }^{18} \mathrm{~F}\right]$-Fluorodeoxyglucose-positron emission tomography for the assessment of histopathologic response and prognosis after completion of neoadjuvant chemotherapy in gastric cancer. J Surg Oncol. 2010;102:135-40.

41. Ott K, Herrmann K, Schuster T, Langer R, Becker K, Wieder HA, et al. Molecular imaging of proliferation and glucose utilization: utility for monitoring response and prognosis after neoadjuvant therapy in locally advanced gastric cancer. Ann Surg Oncol. 2011;18:3316-23. 